\title{
Khảo sát việc dùng dịch thuật như một chiến lược học của người học tiếng Anh tại Cần Tho
}

\section{Investigating the use of translation as a learning strategy of English learners in Can Tho city}

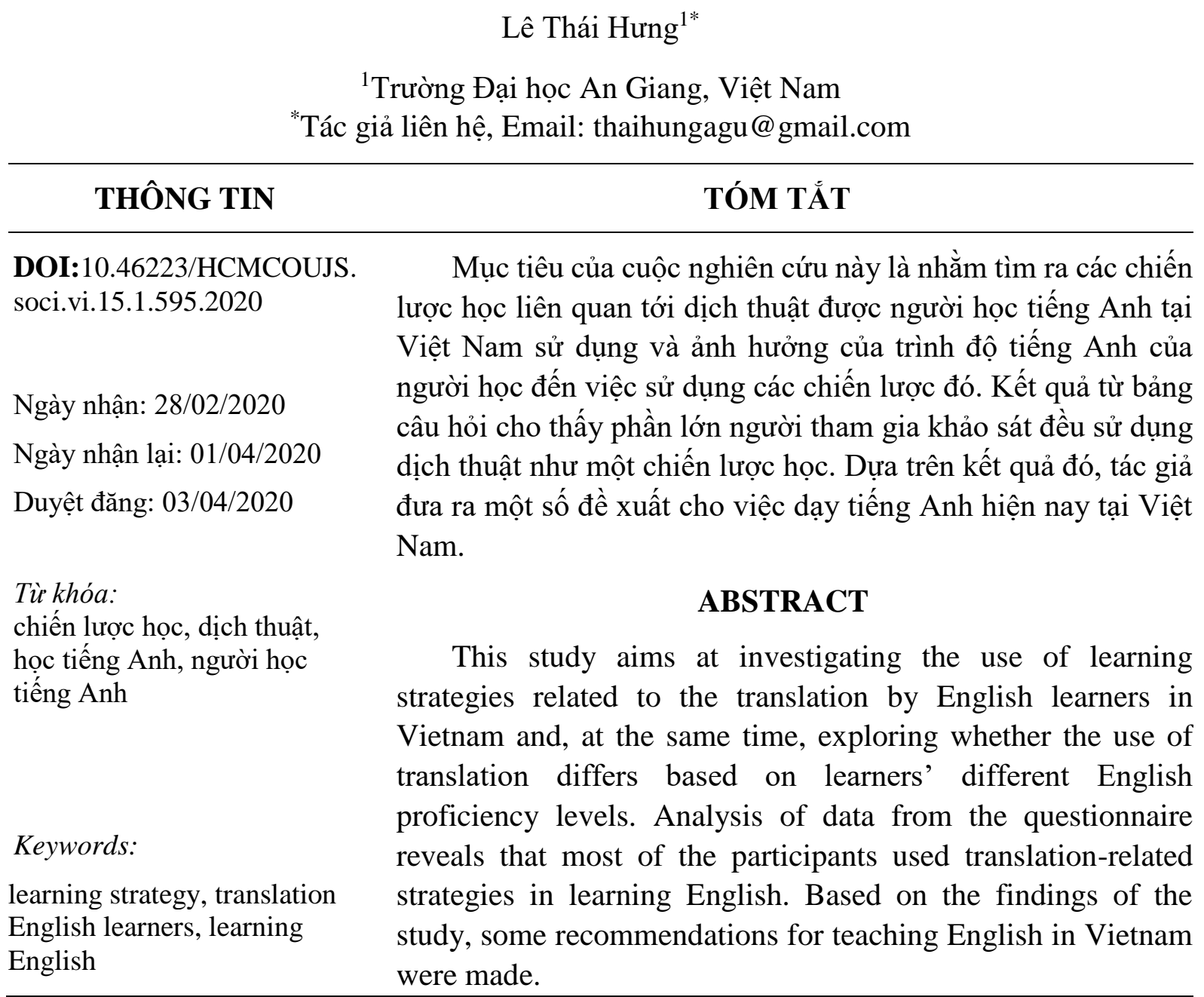

\section{Giới thiệu}

Trong quá trình học ngoại ngữ, cụ thể trong trường hợp này là tiếng Anh, mỗi người học đều tự chọn cho mình những chiến lược học thích hợp. Yang (1999) đã khẳng định rằng chiến lược học tiếng Anh thích hợp sẽ giúp người học có trách nhiệm với việc học của mình và có thể dẫn đến thành công trong sử dụng tiếng Anh. Là một trong số các chiến lược nhận thức, dịch thuật là cách học được không ít người quan tâm hiện nay. Theo đó, khi sử dụng chiến lược này, ngôn ngữ mẹ đẻ được dùng như một kênh trung gian giúp người học tận dụng ngôn ngữ của mình để hiểu một bài đọc và hoạch định trước khi viết ra bằng ngôn ngữ đang học. 


\section{Lược khảo tài liệu}

Dịch thuật được định nghĩa trong từ điển Oxford (2004) là quá trình dịch các từ hay văn bản từ một ngôn ngữ này sang một ngôn ngữ khác. Chiến lược học liên quan đến dịch thuật là "việc dùng ngôn ngữ thứ nhất như là nền tảng để hiểu và/hoặc thực hành ngôn ngữ thứ hai" (Tan, 2015, p. 56).

Trên thực tế, có những giáo viên cũng như nhà nghiên cứu tỏ thái độ tiêu cực đối với việc dùng chiến lược này, trong khi số khác thì lại đề cao tầm quan trọng của nó trong việc dạy và học ngoại ngữ. Nằm trong số những người ủng hộ, Scheffler (2013) cho rằng việc đánh giá lại chiến lược học dựa trên dịch thuật đã được quan tâm từ thế kỷ 21 và đồng thời xem nó như một hoạt động thú vị và hữu ích. Bên cạnh đó, Tan (2015) nhận định "thật là tùy tiện khi phủ nhận hoàn toàn tác động tích cực của việc dùng tiếng mẹ đẻ khi học tiếng Anh" (p.59). Fernández-Guerra (2014) thì đưa ra kết luận người học có thái độ tích cực đối với chiến lược học liên quan đến dịch thuật và tiềm năng của phương pháp này khi họ nhận ra rằng nó giúp cải thiện bốn kỹ năng ngôn ngữ của họ. Còn theo Mollaei, Taghinezhad, và Sadighi (2017) thì đây là một chiến lược dạy và học quan trọng bởi những lí do sau:

- Nó giúp ích trong việc dở bỏ những rào cản tâm lý của người học gặp phải trong môi trường học;

- Nó rất hữu dụng khi dạy các cấu trúc ngữ pháp phức tạp, trừ trường hợp người học đã đủ trình độ để hiểu khi được giải thích bằng ngoại ngữ;

- Nó là một kỹ thuật sẵn có tức thì trong trường hợp tất cả các kỹ thuật thực tiễn khác đều thất bại, chẳng hạn như học thuộc lòng các từ khó;

- Trong hoạt động đọc, khi học viên không hiểu được bài học (do các yếu tố văn hóa), giáo viên có thể áp dụng dịch thuật.

Có nhiều nhà nghiên cứu cũng đã tìm hiểu tác động của việc dùng chiến lược dịch đối với người học tiếng Anh. Karimian và Talebinejad (2013) qua khảo sát đã nhận thấy chiến lược dịch thuật giúp ích cho người học tiếng Anh và tin rằng nó là một chiến lược học quan trọng. Trong một nghiên cứu khác, Mutlu, Bayram, và Demirbüken (2015) khẳng định người học dùng chiến lược dịch thuật trong khi làm bài tập viết và đọc tiếng Anh vì họ cho rằng sẽ dễ dàng hơn khi họ nghĩ cách hoàn thành bài tập bằng tiếng mẹ đẻ trước rồi mới dịch sang tiếng Anh. Chiến lược này khiến họ thấy tự tin hơn. Ngoài ra, từ kết quả nghiên cứu của mình, Ramachandran và Rahimi (2004) cho rằng dịch thuật có thể được dùng như một phương pháp để mở rộng vốn từ vựng của người học. Theo đó, chiến lược dạy và học liên quan đến dịch thuật giúp người học so sánh, đối chiếu hai ngôn ngữ với nhau và từ đó họ phát triển kỹ năng đọc cũng như vốn từ của mình. Khái quát hơn, Dagiliené (2012) thực hiện cuộc nghiên cứu về khả năng giúp người học phát triển các kỹ năng ngôn ngữ như viết, nói, từ vựng, ngữ pháp và đọc. Và cuộc nghiên cứu cho kết quả rất tích cực.

\section{Sự cần thiết của vấn đề nghiên cứu}

Việc tổng hợp tài liệu liên quan cho thấy tính hiệu quả và tầm quan trọng của chiến lược dịch thuật trong việc học ngoại ngữ ở một số nơi trên thế giới. Tuy nhiên, đối với những đối tượng học khác nhau thì tác động của chiến lược này có thể sẽ có sự thay đổi khác nhau, và do 
đó thái độ của người học - đối tượng sử dụng - cũng sẽ khác nhau. Đó là lý do tại sao cần thực hiện nhiều nghiên cứu hơn nữa ở các môi trường học tập khác nhau, các nước khác nhau để thấy được sự đa dạng và tổng quát của việc sử dụng chiến lược này. Trong bối cảnh đó, bài nghiên cứu ra đời nhằm tìm hiểu việc sử dụng chiến lược này tại Việt $\mathrm{Nam}$, cụ thể là tại tỉnh Cần Thơ.

\section{Phương pháp nghiên cứu}

\section{1. Đối tự̣ng nghiên cúu}

Dữ liệu của cuộc nghiên cứu được thu thập từ ý kiến của 178 học sinh khối lớp 12 tại trường PTTH Phan Văn Trị và trường PTTH Châu Thành $\mathrm{A}, \mathrm{Tp}$. Cần Thơ. Các học sinh đều tham gia vào cuộc nghiên cứu trên tinh thần tự nguyện và ý kiến của các em sẽ không ảnh hưởng đến điểm số trên lớp.

\subsection{Phương pháp nghiên cúu và công cụ thu thập dĩ liệu}

Để đạt đến mục tiêu nghiên cứu, một bảng khảo sát được dịch từ "Bảng đánh giá việc dùng dịch thuật như một chiến lược học" của Liao (2006) đã được sử dụng để thu thập dữ liệu. Theo đó, chỉ số độ tin cậy Cronbach's Alpha của bảng khảo sát đã được chứng minh là nằm trong khoảng được chấp nhận là trên 0.70 . Ngoài 3 câu hỏi về thông tin cơ bản, bảng khảo sát còn có 20 câu hỏi về mức độ sử dụng chiến lược dịch thuật, dựa trên thang đo Likert ở 5 mức độ: 1=không bao giờ; $2=$ =rất hiếm khi; $3=$ thỉnh thoảng; 4=thường xuyên; $5=$ luôn luôn.

\subsection{Câu hỏi nghiên cúu}

1. Người học tiếng Anh tại Việt Nam sử dụng các chiến lược liên quan đến dịch thuật ở mức độ nào?

2. Trình độ tiếng Anh của người học có ảnh hưởng đến việc sử dụng chiến lược liên quan đến dịch thuật không?

\section{Kết quả và thảo luận}

\subsection{Mức độ sử dụng chiến lực học tiếng Anh liên quan đến dịch thuật của học sinh}

\section{Bảng 1}

Mức độ sử dụng chiến lược học liên quan đến dịch thuật của học sinh

\begin{tabular}{|l|c|c|c|c|c|}
\hline & $\mathbf{N}$ & Minimum & Maximum & Mean & Std. Deviation \\
\hline MEAN & 178 & 1.30 & 4.30 & 2.7923 & .56735 \\
\hline Valid N (listwise) & 178 & & & & \\
\hline
\end{tabular}

Nguồn: Kết quả phân tích dữ liệu của nhóm nghiên cứu

Với chỉ số trung bình $\mathrm{M}=2.8$ và độ lệch chuẩn $\mathrm{SD}=0.56$, có thể kết luận rằng trong quá trình học tiếng Anh, nhóm học sinh được khảo sát sử dụng chiến lược liên quan đến dịch thuật ở mức độ trung bình. 


\subsection{Nhóm chiến lực đự̛c sử dụng nhiều nhất và ít nhất}

Giá trị trung bình và độ lệch tiêu chuẩn của các câu trả lời đã được tính toán đối với mỗi lĩnh vực sử dụng dịch thuật như một chiến lược học. Dựa trên kết quả có được từ Frequency Test, có thể thấy 3 chiến lược dịch được nhóm khảo sát sử dụng thường xuyên nhất là "Tôi sử dụng thiết bị dịch tự động để giúp cho việc học tiếng Anh" (M=3.86; SD=0.92), "Tôi ghi nhớ nghĩa của từ mới trong tiếng Anh bằng cách nhớ lại nghĩa của chúng trong tiếng Việt" $(\mathrm{M}=3.16$; $\mathrm{SD}=0.83$ ) và "Tôi ghi nhớ nghĩa của từ mới trong tiếng Anh bằng cách nhớ lại nghĩa của chúng trong tiếng Việt” $(\mathrm{M}=3.12 ; \mathrm{SD}=0.91)$.

Ngược lại, 3 chiến lược dịch ít được chọn nhất là "Tôi nghe hoặc đọc các bản tin bằng tiếng Việt trước để hiểu bản tiếng Anh dễ hơn" $(\mathrm{M}=1.78 ; \mathrm{SD}=0.96)$, "Khi không hiểu điều gì đó trong tiếng Anh, tôi sẽ nhở người khác dịch nó sang tiếng Việt" $(\mathrm{M}=1.92 ; \mathrm{SD}=0.85)$ và "Khi xem chương trình truyền hình hay phim bằng tiếng Anh, tôi dò phụ đề để kiểm tra xem mình hiểu có đúng không" $(\mathrm{M}=2.15 ; \mathrm{SD}=0.91)$.

Các câu hỏi liên quan đến việc dùng chiến lược dịch thuật trong khi làm các bài tập rèn luyện kỹ năng nghe, nói, đọc, viết tiếng Anh đều có chỉ số trung bình khá cao $(\mathrm{M}>2.5)$. Đó là các câu hỏi như: "Khi làm bài tập nghe tiếng Anh, trước tiên tôi dịch các câu nói sang tiếng Việt để hiểu nghĩa của chúng", "Khi tôi quên từ hay cách diễn đạt nào đó trong lúc nói tiếng Anh, tôi dịch từ Việt sang Anh để tiếp tục cuộc trò chuyện", "Khi gặp bài đọc khó, tôi dịch sang tiếng Việt trước trong đầu để hiểu được nghĩa của nó" hay "Khi tập viết bằng tiếng Anh, trước tiên tôi suy nghĩ về đề tài bằng tiếng Việt”.

Ngoài ra, nhóm học sinh được khảo sát cũng dùng chiến lược dịch thuật khi học các thành ngữ mới trong tiếng Anh: “Tôi học các thành ngữ tiếng Anh bằng cách tìm hiểu cách dịch của chúng trong tiếng Việt" $(\mathrm{M}=2.45 ; \mathrm{SD}=0.98)$ và "Tôi dịch sang tiếng Việt các thuật ngữ ngữ pháp như từ loại, thì, và sự hòa hợp trong các cấu trúc để làm rõ vai trò của các thành phần trong câu" $(\mathrm{M}=2.62, \mathrm{SD}=0.86)$.

\subsection{Anh hưởng của trình độ tiếng Anh đến việc sử dụng chiến luộc dịch thuật}

Dựa vào điểm số môn tiếng Anh trên lớp, nhóm học sinh được chia thành nhóm Giỏi (Excellent) với điểm trung bình môn trên 8 và nhóm Khá (Good) với điểm trung bình môn nhỏ hơn 8 . Như vậy, trong khuôn khổ bài nghiên cứu này, những học sinh thuộc nhóm Giỏi được xem như là người học có trình độ tiếng Anh cao hơn những học sinh thuộc nhóm Khá. Để tìm câu trả lời cho câu hỏi nghiên cứu thứ hai về ảnh hưởng của trình độ tiếng Anh của người học đối với việc sử dụng chiến lược liên quan đến dịch thuật không, lệnh independent sample t-test đã được sử dụng. Kết quả từ việc phân tích được thể hiện trong Bảng 2 . Theo đó, những học sinh có trình độ tiếng Anh thấp hơn, nghĩa là những học sinh có học lực khá, có khuynh hướng dùng chiến lược dịch thuật hơn $(\mathrm{M}=2.95)$ so với những học sinh có học lực giỏi $(\mathrm{M}=2.54)$. 


\section{Bảng 2}

Ảnh hưởng của trình độ tiếng Anh đối với việc sử dụng chiến lược dịch

\begin{tabular}{|l|c|c|c|c|c|c|}
\hline GROUPS & $\mathbf{N}$ & \% & MEAN & SD & t/F Value & P \\
\hline Excellent & 102 & 57.3 & 2.54 & 0.37 & 2.31 & $\mathbf{0 . 0 0}^{*}$ \\
\hline Good & 76 & 42.7 & 2.95 & 0.35 & & \\
\hline
\end{tabular}

Nguồn: Kết quả phân tích dữ liệu của nhóm nghiên cứu

\subsection{Thảo luận}

Việc nhóm học sinh được khảo sát dùng các chiến lược liên quan đến dịch thuật ở mức độ trung bình cho thấy có sự hạn chế trong việc chọn các chiến lược này. Nói cách khác, họ chưa chủ động sử dụng các chiến lược tốt nhất và toàn diện nhất trong quá trình học tiếng Anh.

Kết quả khảo sát cũng cho thấy người học phần lớn dựa vào công cụ dịch tự động để giúp mình học tiếng Anh. Điều này phản ánh xu hướng học tập nói chung, học ngoại ngữ nói riêng trong thời đại khoa học công nghệ phát triển hiện nay. Công nghệ đang tăng dần sức ảnh hưởng đối với mọi mặt trong đời sống hàng ngày, trong đó có giáo dục. Do đó, cần phải có những nghiên cứu sâu hơn về sức ảnh hưởng của công nghệ thông tin đối với việc học tiếng Anh tại Việt Nam hiện nay.

Kết quả khảo sát còn cho thấy người học cũng dùng dịch thuật trong lúc học 4 kỹ năng ngôn ngữ cùng với các phạm trù khác của ngôn ngữ như từ vựng, ngữ pháp và thành ngữ. Kết quả này trùng khớp với kết luận từ cuộc nghiên cứu của Liao (2006) là người học thường xuyên nhất là dùng dịch thuật để học từ vựng, thành ngữ, ngữ pháp, cũng như để đọc, viết, nói và kiểm tra việc nghe hiểu. Kết quả từ cuộc nghiên cứu của Liao (2006) đã cho thấy các đối tượng tham gia khảo sát tán thành ý kiến cho rằng chiến lược dạy và học liên quan đến dịch thuật đóng vai trò tích cực trong quá trình học tiếng Anh của họ. Họ tin rằng chiến lược dịch thuật giúp họ phát triển các kỹ năng đọc, viết, nói cũng như mở rộng vốn từ, cụm từ và thành ngữ trong quá trình học tiếng Anh. Kết luận này cũng giống kết luận của các nhà nghiên cứu khác như Calis và Dikilitas (2012), Dagiliené (2012). Ngoài ra, những học sinh trong nhóm khảo sát còn cho biết họ dùng dịch thuật để làm rõ sự giống và khác nhau giữa tiếng Việt và tiếng Anh và nếu họ không hiểu một vấn đề nào đó trong tiếng Anh, họ sẽ nhờ người khác giải thích bằng cách dịch lại. Như vậy, chiến lược dịch thuật vẫn được sử dụng rộng rãi để học và hiểu tiếng Anh tại Việt Nam.

Phân tích nhóm 3 chiến lược ít được nhóm người khảo sát chọn nhất cho thấy người học tiếng Anh còn chưa chủ động tự học, chưa tận dụng hết các cơ hội để nâng cao trình độ tiếng Anh của mình. Để khắc phục tình trạng này, trước hết, giảng viên cần xây dựng được thái độ học tập ngoại ngữ tích cực cho sinh viên, qua đó góp phần xây dựng hành vi học ngoại ngữ, đặc biệt là quá trình tự học. Người học cần nhận được những hướng dẫn cụ thể làm thế nào xây dựng ý thức tự học để tự mình tích lũy thêm những kiến thức cần thiết thay vì ngồi chờ đợi giảng viên truyền đạt kiến thức.

Mối tương quan giữa trình độ của người học và việc sử dụng chiến lược dịch đã được nhiều nghiên cứu trước đây đề cập đến. Trùng khớp với kết quả của cuộc nghiên cứu hiện tại, 
nghiên cứu của Husain (1995) đã cho thấy những người mới bắt đầu học phụ thuộc hoàn toàn vào tiếng mẹ đẻ trong suốt quá trình học tiếng Anh, trong khi đó, người học tiếng Anh ở trình độ cao hơn lại không thích sử dụng chiến lược dịch. Tuy nhiên, dựa vào kết quả nghiên cứu của mình, Titford (1985) lại nhận định dịch thuật là một công cụ giải quyết vấn đề cho người học ở cấp độ cao. Đó là bởi họ nhận thức được sự giống và khác nhau giữa hai ngôn ngữ, cho nên họ nhận được nhiều ích lợi từ việc sử dụng cả hai ngôn ngữ cùng lúc một cách có ý thức.

\section{Kết luận và kiến nghị}

Mục tiêu của cuộc nghiên cứu là tìm hiểu việc sử dụng chiến lược học liên quan đến dịch thuật của người học tiếng Anh và có hay không sự khác biệt trong việc sử dụng các chiến lược này giữa những người học có trình độ tiếng Anh khác nhau. Kết quả cho thấy đa số người học dùng các chiến lược liên quan đến dịch thuật và thấy được ích lợi của chúng trong quá trình học bốn kỹ năng nghe, nói, đọc, viết. Hơn nữa, cuộc nghiên cứu còn tái khẳng định việc người học có trình độ tiếng Anh cao sẽ ít dùng chiến lược dịch thuật hơn.

Cuối cùng, từ việc người học ở trình độ tiếng Anh thấp thường dùng chiến lược dịch trong lớp học đã khiến chiến lược này trở thành một phần không thể thiếu trong quá trình học tiếng Anh. Thêm vào đó, việc dịch từ tiếng mẹ đẻ sang tiếng Anh hoặc ngược lại giúp học tăng khả năng xây dựng câu trong tiếng Anh thông qua việc nhớ lại các cấu trúc ngữ pháp được lặp đi lặp lại nhiều lần. Kết quả cuộc nghiên cứu cũng là một minh chứng phản bác những ý kiến chủ quan xem nhẹ vai trò hỗ trợ của dịch thuật trong quá trình học ngoại ngữ, đặc biệt là trong giai đoạn đầu.

Tóm lại, kết quả từ cuộc nghiên cứu này góp một phần vào sự hiểu biết chung về việc sử dụng dịch thuật như một chiến lược học tiếng Anh tại Việt Nam hiện nay. Đã quá rõ ràng, việc dùng dịch thuật trong quá trình học ngoại ngữ không phải là phương pháp lỗi thời như một số nhận định chủ quan. Trái lại, đó vẫn là một người bạn đồng hành đáng tin cậy của người học ngoại ngữ, đặc biệt là khi họ còn đang ở trình độ thấp.

Ngoài ra, cuộc nghiên cứu này còn cho thấy một số kiến nghị đối với việc dạy và học tiếng Anh hiện nay. Giống với kết quả từ các cuộc nghiên cứu ở các nước khác, kết quả cuộc nghiên cứu này cho thấy chiến lược dịch thuật có vai trò không nhỏ trong việc học tiếng Anh tại Việt Nam. Do đó, dịch thuật (hay tiếng mẹ đẻ) nên được lồng ghép vào từ giai đoạn đầu của quá trình học tiếng Anh của người học để giúp họ hiểu bài dễ hơn. Hơn nữa, giáo viên cần phân phối thêm thời gian dịch thuật trong khi dạy bốn kỹ năng, từ vựng, ngữ pháp và thành ngữ, đặc biệt là đối với người mới bắt đầu học. Trên hết, chiến lược dịch thuật cần được xem là một phương pháp hay kỹ thuật dạy và học ngoại ngữ.

Thêm vào đó, nếu giáo viên dùng dịch thuật như một kỹ thuật trong lớp học tiếng Anh để tăng năng lực ngôn ngữ của học viên, thì nó phải được dùng một cách khéo léo, có chủ đích và có hệ thống tùy theo đối tượng tiếp nhận.

Chiến lược dịch thuật cũng nên được dùng một cách thận trọng. Nói cách khác, giáo viên cần dùng dịch thuật như một công cụ hữu ích nhưng phải tránh lạm dụng phương pháp này. Tang (2002) đề nghị giáo viên không nên dùng tiếng mẹ đẻ quá $10 \%$ trong thời gian của một tiết học. Và khi trình độ của học viên càng lên cao thì tỉ lệ này càng nên được thu nhỏ lại. 
Ở một khía cạnh khác, cuộc nghiên cứu này chỉ mang phạm vi hẹp, gói gọn trong một nhóm nhỏ học sinh lớp 12. Ngoài ra, một số yếu tố khác như: giới tính, thái độ của người học đối với chiến lược dịch thuật... cũng không được đưa vào vấn đề nghiên cứu. Bên cạnh đó, thái độ và ý kiến của giáo viên đối với vấn đề này vẫn chưa được đề cập đến. Do đó, cần thiết phải có những nghiên cứu rộng hơn với số lượng người tham gia khảo sát nhiều hơn cùng với những công cụ nghiên cứu phức tạp hơn để có thể đi đến kết luận chính xác và đáng tin cậy hơn về vấn đề này.

\section{Tài liệu tham khảo}

Calis, E., \& Dikilitas, K. (2012). The use of translation in EFL classes as L2 learning practice. Procedia-Social and Behavioral Sciences, 46, 5079-5084.

Dagiliené, I. (2012). Translation as a learning method in English language teaching. Studies About Languages, 21, 124-129. doi:10.5755/j01.sal.0.21.1469

Fernández-Guerra, A. (2014). The usefulness of translation in foreign language learning: Students' attitudes. International Journal of English Language \& Translation Studies, 2(1),153-170.

Hughes, H. (2004). Oxford dictionary of English. Oxford, UK: Oxford University Press.

Husain, K. (1995). Assessing the role of translation as a learning strategy in ESL. International Journal of Translation, 1(2), 59-84.

Karimian, Z., \& Talebinejad, M. R. (2013). Students' use of translation as a learning strategy in EFL classroom. Journal of Language Teaching and Research, 4(3), 605-610.

Liao, P. S. (2006). EFL learners' beliefs about strategy use of translation in English learning. RELC Journal, 37(2), 191-215.

Mollaei, F., Taghinezhad, A., \& Sadighi, F. (2017). Teachers and learners' perceptions of applying translation as a method, strategy, or technique in an Iranian EFL setting. International Journal of Education and Literacy Studies, 5(2), 67-73. doi:10.7575/aiac.ijels.v.5n.2p.6

Mutlu, R. A. G., Bayram, R. A. D., \& Demirbüken, I. B. (2015). Translation as learning strategy of Turkish EFL learners. International Journal on New Trends in Education and their Implications (IJONTE), 6(2), 225-234.

Ramachandran, S. D., \& Rahim, H. A. (2004). Meaning recall and retention: The impact of translation method on elementary level learners' vocabulary learning. RELC Journal, 35(2), 161-178.

Scheffler, P. (2013). Learners' perceptions of grammar-translation as consciousness raising. Language Awareness, 22(3), 255-269. doi:10.1080/09658416.2012.703673

Tan, S. (2015). The relationship between English language proficiency and EFL learners' use of translation strategies. International Journal of Applied Linguistics and Translation, l(4), 55-60.

Tang, J. (2002). Using L1 in the English classroom. English Teaching Forum, 40(1), 36-43. 
Titford, C. (1985). Translation - A post-communicative activity for advanced learners. In C. Titford \& A. E. Hiehe (Eds.), Translation in foreign language teaching and testing (pp. 73-86). Tubingen, Germany: Gunter Narr.

Yang, N. D. (1999). The relationship between EFL learners' beliefs and learning strategy use. System, 27(4), 515-535. doi:10.1016/S0346-251X(99)00048-2 